\title{
Induced vacuum condensates in the background of a singular magnetic vortex in 2+1-dimensional space-time
}

\author{
Yurii A. Sitenko* \\ Bogolyubov Institute for Theoretical Physics, \\ National Academy of Sciences, \\ 14-b Metrologichna str., 252143, Kyiv, Ukraine
}

\begin{abstract}
We show that the vacuum of the quantized massless spinor field in $2+1$ dimensional space-time is polarized in the presence of a singular magnetic vortex. Depending on the choice of the boundary condition at the location of the vortex, either chiral symmetry or parity is broken; the formation of the appropriate vacuum condensates is comprehensively studied. In addition, we find that current, energy and other quantum numbers are induced in the vacuum.
\end{abstract}

PACS numbers: 03.65.Bz, 03.70.+k, 11.10.Kk, 11.30.Rd

Keywords: vacuum condensate, chiral symmetry, singular vortex

\section{Introduction}

Field models in 2+1-dimensional space-time have been intensively explored in recent years. The interest to this subject is motivated by its apparent relevance for the

*Electronic address: yusitenko@bitp.kiev.ua 
description of planar condensed matter systems with rather fascinating properties, including that of high-temperature superconductivity (see, e.g., Refs. [1, 2, 3, 4]). An important role is played by the study of induced vacuum quantum numbers, in particular, induced vacuum condensates exhibiting various symmetry breaking patterns. It has been shown that the homogeneous external magnetic field strength induces the chiral symmetry breaking condensate in the vacuum in the universal manner, i.e. irrespectively of all possible types of interaction among quantized fermion fields [5]. Certain efforts have been undertaken in an attempt to generalize this result to the case of the inhomogeneous external magnetic field strength [6]. The aim of the present paper (see also Ref.[7]) is quite different, being inspired by the famous BohmAharonov effectm 8 - we pose the question: whether the condensate can emerge in the vacuum even in the case of vanishing external magnetic field strength and vanishing interaction among quantized fermion fields? The answer will be positive, and we shall show that the vacuum condensate can be induced by an external field potential rather than an external field strength. Also, all other vacuum polarization effects in the background of the Bohm-Aharonov magnetic field configuration will be determined.

The condensate which describes the pairing of massless fermions and antifermions in the vacuum is conventionally defined as

$$
\mathcal{C}(x)=i\langle\operatorname{vac}|T \bar{\Psi}(x) \Psi(x)| \operatorname{vac}\rangle,
$$

where $\Psi(x)$ is the operator of the second-quantized fermion field and $T$ denotes the time ordering operation. In the background of external classical fields, the vacuum expectation value of the time-ordered product of the fermion field operators takes form

$$
\langle\operatorname{vac}|T \Psi(x) \bar{\Psi}(y)| \operatorname{vac}\rangle=\left\langle x\left|\left(\gamma^{\mu} \nabla_{\mu}\right)^{-1}\right| y\right\rangle
$$

where $\nabla_{\mu}$ is the covariant derivative in this background. Thus, in a static background, condensate (1) is presented as

$$
\mathcal{C}(\mathbf{x})=\operatorname{tr}\left\langle x\left|\gamma^{0}\left(i \partial_{0}-H\right)^{-1}\right| x\right\rangle
$$


where $x=\left(x^{0}, \mathbf{x}\right), \gamma^{\mu}=\left(\gamma^{0}, \gamma\right), \nabla_{\mu}=\left(\partial_{0}, \boldsymbol{\nabla}\right)$ and

$$
H=-i \gamma^{0} \gamma \cdot \nabla
$$

After performing the Wick rotation of the time axis $\left(x^{0}=-i \tau\right)$, Eq.(3) is recast into the form

$$
\mathcal{C}(\mathbf{x})=-\frac{1}{2} \operatorname{tr}\left\langle\mathbf{x}\left|\gamma^{0} \operatorname{sgn}(H)\right| \mathbf{x}\right\rangle
$$

where

$$
\operatorname{sgn}(u)=\left\{\begin{array}{cc}
1, & u>0 \\
-1, & u<0
\end{array}\right\}
$$

Since Hamiltonian $H(4)$ anticommutes with matrix $\gamma^{0}$,

$$
\left[H, \gamma^{0}\right]_{+}=0
$$

one could anticipate that condensate (5) vanishes. However, this might not be the case for some specific background field configurations.

It is instructive to rewright Eq.(5) as

$$
\mathcal{C}(\mathbf{x})=\frac{i}{4} \nabla \cdot \operatorname{tr}\left\langle\left.\mathbf{x}|\gamma| H\right|^{-1} \mid \mathbf{x}\right\rangle
$$

Although all $\gamma$-matrices are traceless, current

$$
\mathcal{J}(\mathbf{x})=\frac{i}{4} \operatorname{tr}\left\langle\left.\mathbf{x}|\gamma| H\right|^{-1} \mid \mathbf{x}\right\rangle
$$

can be nonvanishing, then its nonconservation results in the emergence of vacuum condensate $(7)$.

A peculiar feature of the 2+1-dimensional quantum field theory consists in a possibility to define chiral invariant vacuum condensate (see, e.g., Ref.[9])

$$
\mathcal{P}(x)=\frac{i}{2}\left\langle\operatorname{vac}\left|T \bar{\Psi}(x)\left[\gamma^{3}, \gamma^{5}\right]_{-} \Psi(x)\right| \operatorname{vac}\right\rangle,
$$

which, in a static background, is reduced to the form

$$
\mathcal{P}(\mathbf{x})=-\frac{1}{4} \operatorname{tr}\left\langle\mathbf{x}\left|\gamma^{0}\left[\gamma^{3}, \gamma^{5}\right]_{-} \operatorname{sgn}(H)\right| \mathbf{x}\right\rangle=\boldsymbol{\nabla} \cdot \mathcal{I}(\mathbf{x})
$$


where

$$
\mathcal{I}(\mathbf{x})=\frac{i}{8} \operatorname{tr}\left\langle\left.\mathbf{x}\left|\gamma\left[\gamma^{3}, \gamma^{5}\right]_{-}\right| H\right|^{-1} \mid \mathbf{x}\right\rangle
$$

Here, $\gamma^{3}$ is the $\gamma$-matrix corresponding to the missing $\left(x^{3}\right)$ dimension and

$$
\gamma^{5}=i \gamma^{0} \gamma^{1} \gamma^{2} \gamma^{3}
$$

This vacuum condensate is directly related to the vacuum spin and breaks parity.

In the present paper we consider classical static magnetic field in flat $2+1$ dimensional space-time, as an external background. Thus, the covariant derivative takes form

$$
\nabla=\partial-i \mathbf{V}(\mathbf{x})
$$

where $\mathbf{V}(\mathbf{x})$ is the vector potential of the magnetic field. The magnetic field configuration is chosen to be that of a singular vortex placed at the origin of twodimensional space (the Bohm-Aharonov configuration):

$$
\begin{gathered}
V^{1}(\mathbf{x})=-\Phi^{(0)} \frac{x^{2}}{\left(x^{1}\right)^{2}+\left(x^{2}\right)^{2}}, \quad V^{2}(\mathbf{x})=\Phi^{(0)} \frac{x^{1}}{\left(x^{1}\right)^{2}+\left(x^{2}\right)^{2}}, \\
\boldsymbol{\partial} \times \mathbf{V}(\mathbf{x})=2 \pi \Phi^{(0)} \delta(\mathbf{x})
\end{gathered}
$$

where $\Phi^{(0)}$ is the vortex flux in $2 \pi$ units, i.e. in the London $\left(2 \pi \hbar c e^{-1}\right)$ units, since we use conventional units $\hbar=c=1$ and coupling constant $e$ is included into vector potential $\mathbf{V}(\mathbf{x})$. Evidently, vector potential (14) is undefined at the origin, i.e. the limiting value $\lim _{|\mathbf{x}| \rightarrow 0} \mathbf{V}(\mathbf{x})$ does not exist. Therefore, point $\mathbf{x}=0$ is excluded, and a certain boundary condition has to be imposed at this puncture. Note that topology of the punctured plane is characterized by winding number: $\pi_{1}=\mathbb{Z}$ (here $\pi_{1}$ is the first homotopy group and $\mathbb{Z}$ is the set of integer numbers).

Hamiltonian (4) in the background of singular magnetic vortex (14)-(15) takes form

$$
H=-i \gamma^{0} \gamma_{r} \partial_{r}-i r^{-1} \gamma^{0} \gamma_{\varphi}\left(\partial_{\varphi}-i \Phi^{(0)}\right)
$$

where

$$
\gamma_{r}=\gamma^{1} \cos \varphi+\gamma^{2} \sin \varphi, \quad \gamma_{\varphi}=-\gamma^{1} \sin \varphi+\gamma^{2} \cos \varphi
$$


and polar coordinates,

$$
r=\sqrt{\left(x^{1}\right)^{2}+\left(x^{2}\right)^{2}}, \quad \varphi=\arctan \left(x^{2} / x^{1}\right),
$$

are introduced.

It is natural to require that the Hamiltonian be self-adjoint operator. Usually, Hamiltonians in singular backgrounds are not essentially self-adjoint, and a possibility of their self-adjoint extension (see, e.g., Refs.[10, 11]) has to be explored. If a solution to this problem is found, then it yields the most general boundary condition at the puncture ${ }^{\text {II) }}$. In the case of Hamiltonian (16), further restrictions are imposed by either parity or chiral symmetry conservation. Having specified the boundary condition at the puncture, one can find all vacuum polarization effects in background (14)-(15).

In the next section we obtain two one-parameter families of boundary conditions at the puncture: one is chiral invariant allowing for parity breaking, and another is parity invariant allowing for chiral symmetry breaking. Chiral symmetry breaking effects are considered in Section 3. Parity breaking effects are considered in Section 4. The absence of the twodimensional anomaly is demonstrated in Section 5. Results are summarized in Section 6. Some details in the derivation of the results are outlined in Appendices A and B.

\section{Boundary condition at the location of a vortex}

In 2+1-dimensional space-time, the Clifford algebra has two inequivalent irreducible representations which can be differed in the following way:

$$
i \gamma^{0} \gamma^{1} \gamma^{2}=s, \quad s= \pm 1
$$

\footnotetext{
${ }^{1)}$ The cases of various nonrelativistic Hamiltonians in singular backgrounds are reviewed extensively in monograph 11]. The cases of the massive and massless relativistic threedimensional Hamiltonians in the background of a singular magnetic monopole are considered in Refs. 12, 13, 14, 15. The case of the massive relativistic twodimensional Hamiltonian in background (14)-(15) is considered in Refs. 116, 17, 18, 19].
} 
Choosing matrix $\gamma^{0}$ to be diagonal

$$
\gamma^{0}=\sigma_{3}
$$

one gets the most general form

$$
\gamma^{1}=e^{\frac{i}{2} \sigma_{3} \chi} i \sigma_{1} e^{-\frac{i}{2} \sigma_{3} \chi}, \quad \gamma^{2}=e^{\frac{i}{2} \sigma_{3} \chi} i s \sigma_{2} e^{-\frac{i}{2} \sigma_{3} \chi},
$$

where $\sigma_{1}, \sigma_{2}$ and $\sigma_{3}$ are the Pauli matrices, and $\chi$ is the parameter varying in the interval $0 \leq \chi<2 \pi$ to go over to the equivalent representations. Since the algebra of the Pauli matrices is complete, there is no any other $2 \times 2$ matrix which anticommutes with the above $\gamma$-matrices. Therefore, in order to generate chiral symmetry transformation, one has to consider a reducible $4 \times 4$ representation composed as a direct sum of two inequivalent irreducible $2 \times 2$ ones (see, e.g., Ref. [9]). Taking into account Eqs.(19) and (20), we get the most general form of the $4 \times 4 \gamma$-matrices:

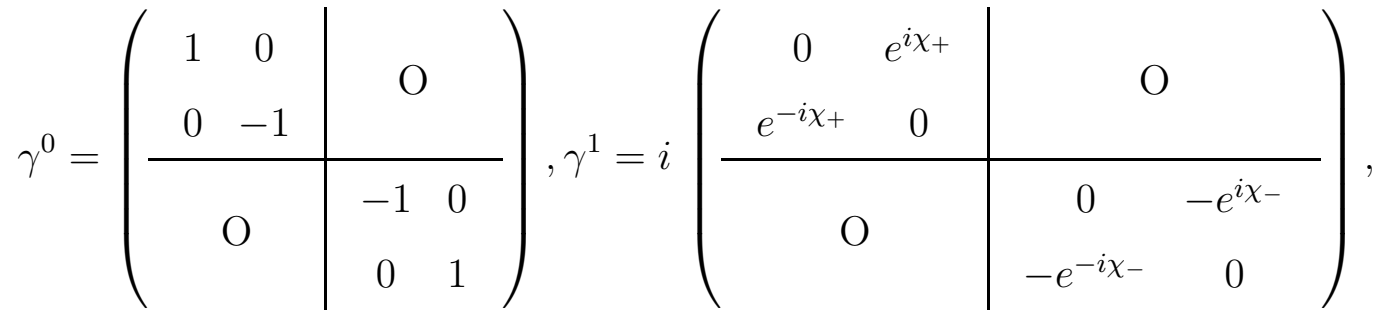

$$
\begin{aligned}
& \gamma^{2}=s\left(\begin{array}{cc|cc}
0 & e^{i \chi_{+}} & \mathrm{O} \\
-e^{-i \chi_{+}} & 0 & & \\
\hline \mathrm{O} & \begin{array}{cc}
0 & -e^{i \chi_{-}} \\
e^{-i \chi_{-}} & 0
\end{array}
\end{array}\right),
\end{aligned}
$$

where $0 \leq \chi_{ \pm}<2 \pi$. The algebra is completed by adding

$$
\begin{aligned}
& \gamma^{3}=i s\left(\begin{array}{c|cc}
\mathrm{O}^{\frac{i}{2}\left(\chi_{+}-\chi_{-}\right)} & 0 \\
0 & 0 & e^{-\frac{i}{2}\left(\chi_{+}-\chi_{-}\right)} \\
\hline e^{-\frac{i}{2}\left(\chi_{+}-\chi_{-}\right)} & 0 \\
0 & e^{\frac{i}{2}\left(\chi_{+}-\chi_{-}\right)} & \multicolumn{2}{|c}{\mathrm{O}}
\end{array}\right) \text {, }
\end{aligned}
$$

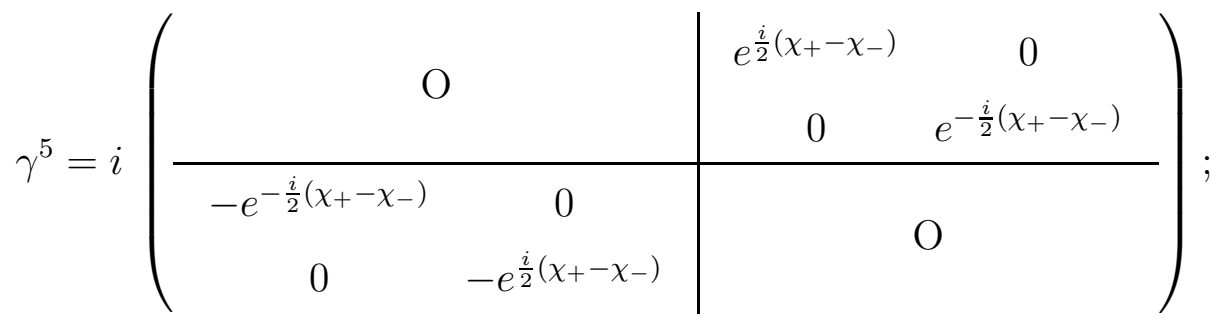


note that the representation which is mostly used (see Ref. [9]) corresponds to $s=1$ and $\chi_{+}=\chi_{-}=0$. Note also that

$$
\frac{1}{2}\left[\gamma^{3}, \gamma^{5}\right]_{-}=s\left(\begin{array}{cc|cc}
1 & 0 & 0 \\
0 & 1 & & \\
\hline 0 & -1 & 0 \\
0 & -1
\end{array}\right)
$$

is sometimes called as the $\tau_{3}$-matrix (see, e.g., Ref.[2]).

One can define the parity transformation

$$
\Psi\left(x^{0}, x^{1}, x^{2}\right) \rightarrow i \gamma^{2} \gamma^{3} \Psi\left(x^{0}, x^{1},-x^{2}\right)
$$

and the chiral symmetry transformation

$$
\Psi\left(x^{0}, x^{1}, x^{2}\right) \rightarrow e^{i \omega \gamma^{5}} \Psi\left(x^{0}, x^{1}, x^{2}\right)
$$

as well as transformations with matrix $\gamma^{5}$ replaced by $i \gamma^{3}, \frac{1}{2}\left[\gamma^{3}, \gamma^{5}\right]_{-}$and the unity matrix.

Using Eq.(21), Hamiltonian (16) is recast into the form

$$
H=\left(\begin{array}{cc}
H_{+} & 0 \\
0 & H_{-}
\end{array}\right)
$$

where

$$
H_{ \pm}=\left(\begin{array}{cc}
0 & e^{i \chi_{ \pm}-i s \varphi}\left[\partial_{r}-r^{-1}\left(i \partial_{\varphi}+\Phi^{(0)}\right)\right] \\
e^{-i \chi_{ \pm}+i s \varphi}\left[-\partial_{r}-r^{-1}\left(i \partial_{\varphi}+\Phi^{(0)}\right)\right] & 0
\end{array}\right) .
$$

Equation of motion

$$
\left(i \partial_{0}-H\right) \Psi(x)=0
$$

is invariant under the chiral symmetry transformation (as well as under transformations generated by $i \gamma^{3}$ and $\left.\frac{1}{2}\left[\gamma^{3}, \gamma^{5}\right]_{-}\right)$. A single-valued solution to Eq.(28) is presented conventionally as

$$
\Psi(x)=\sum_{n \in \mathbb{Z}} \int_{0}^{\infty} d E E e^{-i E x^{0}}<\mathbf{x}\left|E, n>a_{E n}+\sum_{n \in \mathbb{Z}} \int_{0}^{-\infty} d E E e^{-i E x^{0}}<\mathbf{x}\right| E, n>b_{E n}^{+},
$$


where $a_{E n}^{+}$and $a_{E n}\left(b_{E n}^{+}\right.$and $\left.b_{E n}\right)$ are the fermion (antifermion) creation and annihilation operators satisfying anticommutation relations

$$
\left[a_{E n}, a_{E^{\prime} n^{\prime}}^{+}\right]_{+}=\left[b_{E n}, b_{E^{\prime} n^{\prime}}^{+}\right]_{+}=\frac{\delta\left(E-E^{\prime}\right)}{\sqrt{\left|E E^{\prime}\right|}} \delta_{n n^{\prime}},
$$

and

$$
\langle\mathbf{x} \mid E, n\rangle=\left(\begin{array}{c}
f_{n}^{+}(r, E) e^{i n \varphi} \\
g_{n}^{+}(r, E) e^{i(n+s) \varphi} \\
f_{n}^{-}(r, E) e^{i n \varphi} \\
g_{n}^{-}(r, E) e^{i(n+s) \varphi}
\end{array}\right)
$$

radial functions $f_{n}^{ \pm}$and $g_{n}^{ \pm}$satisfy the system of equations

$$
\begin{gathered}
e^{-i \chi_{ \pm}}\left[-\partial_{r}+s\left(n-\Phi^{(0)}\right) r^{-1}\right] f_{n}(r, E)=E g_{n}(r, E), \\
e^{i \chi_{ \pm}}\left[\partial_{r}+s\left(n-\Phi^{(0)}+s\right) r^{-1}\right] g_{n}(r, E)=E f_{n}(r, E) .
\end{gathered}
$$

Decomposing the value of the vortex flux into the integer and fractional parts,

$$
\Phi^{(0)}=\left[\left[\Phi^{(0)}\right]\right]+\left\{\left|\Phi^{(0)}\right|\right\}, \quad 0 \leq\left\{\left|\Phi^{(0)}\right|\right\}<1
$$

([[u]] denotes the integer part of quantity $u$ ), one can note that the case of $\left\{\left|\Phi^{(0)}\right|\right\}=0$ is equivalent to the case of trivial topology, i.e. absence of the vortex $\left(\Phi^{(0)}=0\right) \cdot$. $^{2}$ In the case of $0<\left\{\left|\Phi^{(0)}\right|\right\}<1$ the condition of regularity at the puncture $r=0$ can be imposed on the modes with $n \neq n_{0}$ only, where

$$
n_{0}=\left[\left[\Phi^{(0)}\right]\right]+\frac{1}{2}-\frac{1}{2} s
$$

consequently, we get

$$
\begin{gathered}
\left(\begin{array}{c}
f_{n}^{ \pm} \\
g_{n}^{ \pm}
\end{array}\right)=\frac{1}{2 \sqrt{\pi}}\left(\begin{array}{c}
J_{l-F}(k r) e^{i \chi_{ \pm}} \\
\operatorname{sgn}(E) J_{l+1-F}(k r)
\end{array}\right), \quad l=s\left(n-n_{0}\right)>0 \\
\left(\begin{array}{c}
f_{n}^{ \pm} \\
g_{n}^{ \pm}
\end{array}\right)=\frac{1}{2 \sqrt{\pi}}\left(\begin{array}{c}
J_{l^{\prime}+F}(k r) e^{i \chi_{ \pm}} \\
-\operatorname{sgn}(E) J_{l^{\prime}-1+F}(k r)
\end{array}\right), \quad l^{\prime}=s\left(n_{0}-n\right)>0
\end{gathered}
$$

\footnotetext{
${ }^{2)}$ This confirms once more the general fact that a singular magnetic vortex is physically unob-
} servbale at integer values of the vortex flux [8]. It was as far back as 1931 that Dirac used actually this fact to obtain his remarkable condition for the magnetic monopole quantization [20]. 
here $k=|E|, J_{\rho}(u)$ is the Bessel function of order $\rho$, and

$$
F=s\left\{\left|\Phi^{(0)}\right|\right\}+\frac{1}{2}-\frac{1}{2} s
$$

Thus, the partial Hamiltonians corresponding to $n \neq n_{0}$ are essentially selfadjoint when defined on the domain of regular functions, and that is only the partial Hamiltonian corresponding to $n=n_{0}$ that needs a self-adjoint extension. The method of self-adjoint extensions (see, e.g., Refs. 10, 11]) is employed in Appendix A, resulting in a boundary condition which entails the irregular behaviour of the mode with $n=n_{0}$ at the puncture $r=0$. Actually, there are two different possibilities to choose a physically reasonable boundary condition: one is chiral invariant and parity violating, while another is parity invariant and chiral symmetry violating. The chiral invariant choice is

$$
\cos \left(s \frac{\Theta}{2}+\frac{\pi}{4}\right) \lim _{r \rightarrow 0}(\mu r)^{F} f_{n_{0}}^{ \pm}=-e^{i \chi_{ \pm}} \sin \left(s \frac{\Theta}{2}+\frac{\pi}{4}\right) \lim _{r \rightarrow 0}(\mu r)^{1-F} g_{n_{0}}^{ \pm},
$$

then the irregular mode takes form

$$
\begin{gathered}
\left(\begin{array}{c}
f_{n_{0}}^{ \pm} \\
g_{n_{0}}^{ \pm}
\end{array}\right)=\frac{1}{2 \sqrt{\pi\left[1+\sin \left(2 \nu_{E}\right) \cos (F \pi)\right]}} \times \\
\times\left(\begin{array}{c}
{\left[\sin \left(\nu_{E}\right) J_{-F}(k r)+\cos \left(\nu_{E}\right) J_{F}(k r)\right] e^{i \chi_{ \pm}}} \\
\operatorname{sgn}(E)\left[\sin \left(\nu_{E}\right) J_{1-F}(k r)-\cos \left(\nu_{E}\right) J_{-1+F}(k r)\right]
\end{array}\right) .
\end{gathered}
$$

The parity invariant choice is

$$
\cos \left(s \frac{\Theta}{2}+\frac{\pi}{4}\right) \lim _{r \rightarrow 0}(\mu r)^{F} f_{n_{0}}^{ \pm}=\mp e^{i \chi_{ \pm}} \sin \left(s \frac{\Theta}{2}+\frac{\pi}{4}\right) \lim _{r \rightarrow 0}(\mu r)^{1-F} g_{n_{0}}^{ \pm},
$$

then the irregular mode takes form

$$
\begin{gathered}
\left(\begin{array}{c}
f_{n_{0}}^{ \pm} \\
g_{n_{0}}^{ \pm}
\end{array}\right)=\frac{1}{2 \sqrt{\pi\left[1 \pm \sin \left(2 \nu_{E}\right) \cos (F \pi)\right]}} \times \\
\times\left(\begin{array}{c}
{\left[\sin \left(\nu_{E}\right) J_{-F}(k r) \pm \cos \left(\nu_{E}\right) J_{F}(k r)\right] e^{i \chi \pm}} \\
\operatorname{sgn}(E)\left[\sin \left(\nu_{E}\right) J_{1-F}(k r) \mp \cos \left(\nu_{E}\right) J_{-1+F}(k r)\right]
\end{array}\right) .
\end{gathered}
$$


Here, the energy dependent parameter $\left(\nu_{E}\right)$ is expressed through the self-adjoint extension parameter $(\Theta)$ via relation

$$
\tan \left(\nu_{E}\right)=\operatorname{sgn}(E)\left(\frac{k}{2 \mu}\right)^{2 F-1} \frac{\Gamma(1-F)}{\Gamma(F)} \tan \left(s \frac{\Theta}{2}+\frac{\pi}{4}\right)
$$

$\mu>0$ is the parameter of the dimension of mass, which is introduced just to scale different irregular behaviours of the $f$ and $g$ components, and $\Gamma(u)$ is the Euler gamma function; note that, since Eqs.(38) and (40) are periodic in $\Theta$ with period $2 \pi$, all permissible values of $\Theta$ can be restricted, without a loss of generality, to range $-\pi \leq \Theta \leq \pi$.

Concluding this section, let us note that conditions (38) and (40) coincide in the case of

$$
\cos \Theta=0 \text {, }
$$

due to vanishing of either their left-hand sides (at $\Theta=s \frac{\pi}{2}$ ) or their right-hand sides (at $\Theta=-s \frac{\pi}{2}$ ). Thus, both parity and chiral symmetry are conserved in the case of Eq.(43).

\section{Chiral symmetry breaking}

Let us consider vacuum polarization effects under the parity invariant condition (40). Using the complete set of solutions (35), (36) and (41), we can evaluate current $\mathcal{J}(\mathbf{x})$ (8). Since angular component

$$
\mathcal{J}_{\varphi}(\mathbf{x})=\frac{i}{4} \operatorname{tr}\left\langle\left.\mathbf{x}\left|\gamma_{\varphi}\right| H\right|^{-1} \mid \mathbf{x}\right\rangle
$$

vanishes, there remains only radial component

$$
\mathcal{J}_{r}(\mathbf{x})=\frac{i}{4} \operatorname{tr}\left\langle\left.\mathbf{x}\left|\gamma_{r}\right| H\right|^{-1} \mid \mathbf{x}\right\rangle
$$

which can be obviously presented as a sum over the upper and lower inequivalent irreducible representations:

$$
\mathcal{J}_{r}(\mathbf{x})=\mathcal{J}_{r}^{+}(\mathbf{x})+\mathcal{J}_{r}^{-}(\mathbf{x}) .
$$


We find that that the contribution of regular modes (35) and (36) is cancelled,

$$
\left[\mathcal{J}_{r}^{+}(\mathbf{x})\right]_{\mathrm{REG}}=-\left[\mathcal{J}_{r}^{-}(\mathbf{x})\right]_{\mathrm{REG}}
$$

while the contribution of irregular mode (41) survives,

$$
\left[\mathcal{J}_{r}^{+}(\mathbf{x})\right]_{\text {IRREG }}=\left[\mathcal{J}_{r}^{-}(\mathbf{x})\right]_{\text {IRREG }}
$$

consequently, we get

$$
\begin{gathered}
\mathcal{J}_{r}(\mathbf{x})=-\frac{1}{4 \pi} \int_{0}^{\infty} d k\left\{A\left(\frac{k}{\mu}\right)^{2 F-1}\left[L_{(+)}+L_{(-)}\right] J_{-F}(k r) J_{1-F}(k r)+\right. \\
+\left[L_{(+)}-L_{(-)}\right]\left[J_{F}(k r) J_{1-F}(k r)-J_{-F}(k r) J_{-1+F}(k r)\right]- \\
\left.-A^{-1}\left(\frac{k}{\mu}\right)^{1-2 F}\left[L_{(+)}+L_{(-)}\right] J_{F}(k r) J_{-1+F}(k r)\right\}
\end{gathered}
$$

where

$$
A=2^{1-2 F} \frac{\Gamma(1-F)}{\Gamma(F)} \tan \left(s \frac{\Theta}{2}+\frac{\pi}{4}\right)
$$

and

$$
L_{( \pm)}=2^{-1}\left\{\cos (F \pi) \pm \cosh \left[(2 F-1) \ln \left(\frac{k}{\mu}\right)+\ln A\right]\right\}^{-1}
$$

incidentally, one can verify that current $\mathcal{I}(\mathbf{x})$ (11) vanishes. Extending the integrand in Eq.(49) to the complex $k$-plane, using the Cauchy theorem to deform the contour of integration (for more details see Refs. [18, 19]) and introducing the dimensionless integration variable, we recast Eq.(49) into the form

$$
\mathcal{J}_{r}(\mathbf{x})=\frac{\sin (F \pi)}{\pi^{3} r^{2}} \int_{0}^{\infty} d w \frac{K_{F}(w) K_{1-F}(w)}{\cosh \left[(2 F-1) \ln \left(\frac{w}{\mu r}\right)+\ln A\right]},
$$

where

$$
K_{\rho}(w)=\frac{\pi}{2 \sin (\rho \pi)}\left[I_{-\rho}(w)-I_{\rho}(w)\right]
$$

is the Macdonald function of order $\rho\left(I_{\rho}(w)\right.$ is the modified Bessel function of order $\rho)$. Taking into account the invariance of Eq.(52) under $s \rightarrow-s$, we rewrite it as

$$
\mathcal{J}_{r}(\mathbf{x})=\frac{\sin \left(\left\{\left|\Phi^{(0)}\right|\right\} \pi\right)}{\pi^{3} r^{2}} \int_{0}^{\infty} d w \frac{K_{\left.\left\{\Phi^{(0)}\right\}\right\}}(w) K_{\left.1-\left\{\Phi^{(0)}\right\}\right\}}(w)}{\cosh \left[\left(2\left\{\Phi^{(0)}\right\}-1\right) \ln \left(\frac{w}{\mu r}\right)+\ln \bar{A}\right]},
$$


where

$$
\bar{A}=2^{1-2\left\{\Phi^{(0)}\right\}} \frac{\Gamma\left(1-\left\{\left|\Phi^{(0)}\right|\right\}\right)}{\Gamma\left(\left\{\left|\Phi^{(0)}\right|\right\}\right)} \tan \left(\frac{\Theta}{2}+\frac{\pi}{4}\right) .
$$

Finally, taking into account Eqs.(7)-(8) and relations

$$
\mathcal{J}_{\varphi}(\mathbf{x})=0, \quad \boldsymbol{\nabla} \cdot \mathcal{J}(\mathbf{x})=r^{-1} \partial_{r}\left[r \mathcal{J}_{r}(\mathbf{x})\right]
$$

we get the following expression for vacuum condensate (5):

$$
\mathcal{C}(\mathbf{x})=-\frac{\sin \left(\left\{\left|\Phi^{(0)}\right|\right\} \pi\right)}{\pi^{3} r^{2}} \int_{0}^{\infty} d w w \frac{K_{\left\{\Phi^{(0)} \mid\right\}}^{2}(w)+K_{\left.1-\left\{\Phi^{(0)}\right\}\right\}}^{2}(w)}{\cosh \left[\left(2\left\{\left|\Phi^{(0)}\right|\right\}-1\right) \ln \left(\frac{w}{\mu r}\right)+\ln \bar{A}\right]}
$$

Note that the integral in Eq.(53) is ill-defined at half-integer values of the vortex flux (at $\left\{\left|\Phi^{(0)}\right|\right\}=\frac{1}{2}$ ). On the contrary, the integral in Eq.(56) is well defined at all values of the vortex flux. In particular, taking into account relation

$$
\left.\bar{A}\right|_{\left.\left\{\Phi^{(0)}\right\}\right\}=\frac{1}{2}}=\tan \left(\frac{\Theta}{2}+\frac{\pi}{4}\right)
$$

we get

$$
\left.\mathcal{C}(\mathbf{x})\right|_{\left\{\Phi^{(0)}\right\}=\frac{1}{2}}=-\frac{\cos \Theta}{2 \pi^{2} r^{2}}
$$

Since current $\mathcal{J}(\mathbf{x})$ plays a merely supplementary role and the quantity of physical significance is condensate $\mathcal{C}(\mathbf{x})$, one can just pay no attention to a divergence of the integral in Eq.(53) at $\left\{\left|\Phi^{(0)}\right|\right\}=\frac{1}{2}$. However, this divergence reveals itself when considering the total condensate,

$$
\mathcal{C}=\int d^{2} x \mathcal{C}(\mathbf{x})
$$

which can be rewritten as

$$
\mathcal{C}=2 \pi\left\{\left.\left[r \mathcal{J}_{r}(\mathbf{x})\right]\right|_{r=\infty}-\left.\left[r \mathcal{J}_{r}(\mathbf{x})\right]\right|_{r=0}\right\}
$$

Therefore, we show in Appendix B, how regularization with the help of mass parameter $M$ can be introduced consistently. In particular, after the removal of the regularization parameter $(M \rightarrow 0)$ we find

$$
\left.\operatorname{sgn}(\cos \Theta) r \mathcal{J}_{r}(\mathbf{x})\right|_{\left\{\Phi^{(0)}\right\}=\frac{1}{2}}=\infty
$$


We find also that the regularized version of Eq.(60),

$$
\mathcal{C}^{(M)}=2 \pi\left\{\left.\left[r \mathcal{J}_{r}(\mathbf{x} \mid M)\right]\right|_{r=\infty}-\left.\left[r \mathcal{J}_{r}(\mathbf{x} \mid M)\right]\right|_{r=0}\right\}
$$

takes an infinite value at $\left\{\left|\Phi^{(0)}\right|\right\}=\frac{1}{2}$. This value is in accordance with the direct evaluation of total condensate (59) using the explicit form of Eq.(56), which yields:

$$
\mathcal{C}=-\frac{\operatorname{sgn}(\cos \Theta)}{\left|2\left\{\left|\Phi^{(0)}\right|\right\}-1\right|}
$$

Note that all above quantities are certainly vanishing in the case when Eq.(43) holds.

What about other vacuum polarization effects? The vacuum energy density can be defined as

$$
\varepsilon^{\mathrm{ren}}(\mathbf{x})=-\frac{1}{2} \lim _{z \rightarrow-\frac{1}{2}} \lim _{M \rightarrow 0}\left[\zeta_{\mathbf{x}}(z \mid M)-\zeta_{\mathbf{x}}^{(0)}(z \mid M)\right]
$$

where

$$
\zeta_{\mathbf{x}}(z \mid M)=\operatorname{tr}\left\langle\mathbf{x}|| H^{2}+\left.M^{2}\right|^{-z} \mid \mathbf{x}\right\rangle
$$

is the zeta function density, $M$ is the mass parameter which is introduced to regularize the infrared divergence, while the ultraviolet divergence is regularized by means of complex parameter $z$ : functions $\zeta_{\mathbf{x}}(z \mid M)$ and $\zeta_{\mathbf{x}}^{(0)}(z \mid M)$ are evaluated at $\operatorname{Re} z>1$ and then analytically continued to the whole complex $z$-plane (see Refs. 21, 22, 23). Note that the zeta function density in the trivial background takes form

$$
\zeta_{\mathbf{x}}^{(0)}(z \mid M)=\frac{|M|^{2(1-z)}}{\pi(z-1)}
$$

Let us also define the conventional vacuum current,

$$
\mathbf{j}(\mathbf{x})=-\frac{1}{2} \operatorname{tr}\left\langle\mathbf{x}\left|\gamma^{0} \gamma \operatorname{sgn}(H)\right| \mathbf{x}\right\rangle
$$

in particular, its angular component,

$$
j_{\varphi}(\mathbf{x})=-\frac{1}{2} \operatorname{tr}\left\langle\mathbf{x}\left|\gamma^{0} \gamma_{\varphi} \operatorname{sgn}(H)\right| \mathbf{x}\right\rangle
$$

Similarly to the evaluation of condensate (56), we get

$$
\zeta_{\mathbf{x}}(z \mid M)=\frac{|M|^{2(1-z)}}{\pi(z-1)}+\frac{4 \sin \left(\left\{\left|\Phi^{(0)}\right|\right\} \pi\right)}{\pi^{3}} \frac{\sin (z \pi)}{z-1} r^{2(z-1)} \times
$$




$$
\begin{gathered}
\times \int_{|M| r}^{\infty} d w\left(w^{2}-M^{2} r^{2}\right)^{1-z} K_{\left.\left\{\Phi^{(0)}\right\}\right\}}(w) K_{1-F}(w)+ \\
+\frac{2 \sin \left(\left\{\left|\Phi^{(0)}\right|\right\} \pi\right)}{\pi^{3}} \sin (z \pi) r^{2(z-1)} \int_{|M| r}^{\infty} d w w\left(w^{2}-M^{2} r^{2}\right)^{-z}\left\{K_{\left\{\Phi^{(0)}\right\}}^{2}(w)+K_{1-\left\{\mid \Phi^{(0)}\right\}}^{2}(w)+\right. \\
\left.+\left[K_{\left.\left\{\Phi^{(0)}\right\}\right\}}^{2}(w)-K_{\left.1-\left\{\Phi^{(0)}\right\}\right\}}^{2}(w)\right] \tanh \left[\left(2\left\{\left|\Phi^{(0)}\right|\right\}-1\right) \ln \left(\frac{w}{\mu r}\right)+\ln \bar{A}\right]\right\},
\end{gathered}
$$

and, consequently,

$$
\begin{gathered}
\varepsilon^{\mathrm{ren}}(\mathbf{x})=\frac{\sin \left(\left\{\left|\Phi^{(0)}\right|\right\} \pi\right)}{\pi r^{3}}\left\{\frac{\frac{1}{2}-\left\{\left|\Phi^{(0)}\right|\right\}}{6 \cos \left(\left\{\left|\Phi^{(0)}\right|\right\} \pi\right)}\left[\frac{3}{4}-\left\{\left|\Phi^{(0)}\right|\right\}\left(1-\left\{\left|\Phi^{(0)}\right|\right\}\right)\right]+\right. \\
\left.+\frac{1}{\pi^{2}} \int_{0}^{\infty} d w w^{2}\left[K_{\left\{\mid \Phi^{(0)}\right\}}^{2}(w)-K_{1-\left\{\Phi^{(0)}\right\}}^{2}(w)\right] \tanh \left[\left(2\left\{\Phi^{(0)} \mid\right\}-1\right) \ln \left(\frac{w}{\mu r}\right)+\ln \bar{A}\right]\right\} .
\end{gathered}
$$

Also we get

$$
\begin{gathered}
j_{\varphi}(\mathbf{x})=\frac{\sin \left(\left\{\left|\Phi^{(0)}\right|\right\} \pi\right)}{\pi r^{2}}\left\{\frac{\left(\left\{\left|\Phi^{(0)}\right|\right\}-\frac{1}{2}\right)^{2}}{2 \cos \left(\left\{\left|\Phi^{(0)}\right|\right\} \pi\right)}-\right. \\
\left.-\frac{2}{\pi^{2}} \int_{0}^{\infty} d w w K_{\left.\left\{\Phi^{(0)}\right\}\right\}}(w) K_{\left.1-\left\{\Phi^{(0)}\right\}\right\}}(w) \tanh \left[\left(2\left\{\left|\Phi^{(0)}\right|\right\}-1\right) \ln \left(\frac{w}{\mu r}\right)+\ln \bar{A}\right]\right\} .
\end{gathered}
$$

Since the radial component of the vacuum current is not induced, Eqs.(56), (70) and (71) comprise all effects of the vacuum polarization in background (14)-(15) under condition (40). At half-integer values of the vortex flux, Eqs.(70) and (71) take form

$$
\left.\varepsilon^{\mathrm{ren}}(\mathbf{x})\right|_{\left\{\Phi^{(0)}\right\}=\frac{1}{2}}=\frac{1}{12 \pi^{2} r^{3}}
$$

and

$$
\left.j_{\varphi}(\mathbf{x})\right|_{\left.\left\{\Phi^{(0)}\right\}\right\}=\frac{1}{2}}=-\frac{\sin \Theta}{2 \pi^{2} r^{2}} ;
$$

note the $\Theta$ independence of Eq.(72).

We conclude this section by noting that the vacuum polarization effects violate translational invariance but remain invariant with respect to a rotation around the vortex, depending only on the distance from the vortex. At large distances they are decreasing by power law:

$$
\mathcal{C}(\mathbf{x}) \underset{r \rightarrow \infty}{=}-\frac{\sin \left(\left\{\left|\Phi^{(0)}\right|\right\} \pi\right)}{\pi^{2} r^{2}} \times
$$




$$
\begin{gathered}
\times\left\{\begin{array}{c}
(\mu r)^{2\left\{\mid \Phi^{(0)}\right\}-1} \bar{A}^{-1} \frac{\Gamma\left(\frac{3}{2}-\left\{\left|\Phi^{(0)}\right|\right\}\right) \Gamma\left(\frac{3}{2}-2\left\{\Phi^{(0)}\right\}\right)}{\Gamma\left(1-\left\{\mid \Phi^{(0)}\right\}\right)}, \quad 0<\left\{\mid \Phi^{(0)}\right\}<\frac{1}{2} \\
(\mu r)^{1-2\left\{\left\{\Phi^{(0)}\right\}\right.} \bar{A} \frac{\Gamma\left(\left\{\Phi^{(0)}\right\}+\frac{1}{2}\right) \Gamma\left(2\left\{\Phi^{(0)}\right\}-\frac{1}{2}\right)}{\Gamma\left(\left\{\Phi^{(0)}\right\}\right)}, \quad \frac{1}{2}<\left\{\left|\Phi^{(0)}\right|\right\}<1
\end{array}\right. \\
\varepsilon^{\mathrm{ren}}(\mathbf{x}) \underset{r \rightarrow \infty}{=} \frac{\tan \left(\left\{\left|\Phi^{(0)}\right|\right\} \pi\right)}{2 \pi r^{3}}\left(\left\{\left|\Phi^{(0)}\right|\right\}-\frac{1}{2}\right) \times \\
\times\left[\frac{1}{3}\left\{\left|\Phi^{(0)}\right|\right\}\left(1-\left\{\left|\Phi^{(0)}\right|\right\}\right)-\frac{1}{4}+\frac{1}{2}\left|\left\{\left|\Phi^{(0)}\right|\right\}-\frac{1}{2}\right|\right] \\
j_{\varphi}(\mathbf{x}) \underset{r \rightarrow \infty}{=} \frac{\tan \left(\left\{\left|\Phi^{(0)}\right|\right\} \pi\right)}{2 \pi r^{2}}\left|\left\{\left|\Phi^{(0)}\right|\right\}-\frac{1}{2}\right|\left(\left|\left\{\left|\Phi^{(0)}\right|\right\}-\frac{1}{2}\right|-1\right) .
\end{gathered}
$$

\section{$4 \quad$ Parity breaking}

Let us consider vacuum polarization effects under the chiral invariant condition (38). Using the complete set of solutions (35), (36) and (39), we find immediately

$$
\mathcal{J}_{r}^{+}(\mathbf{x})=-\mathcal{J}_{r}^{-}(\mathbf{x})
$$

and, consequently, current $\mathcal{J}(\mathbf{x})$ (8) vanishes. Concerning current $\mathcal{I}(\mathbf{x})(11)$, we find

$$
\mathcal{I}_{\varphi}(\mathbf{x})=0
$$

and

$$
\mathcal{I}_{r}^{+}(\mathbf{x})=\mathcal{I}_{r}^{-}(\mathbf{x})
$$

The contribution of regular solutions (35) and (36) is cancelled upon summation

over the sign of energy, while the contribution of irregular solution (39) survives, resulting in

$$
\mathcal{I}_{r}(\mathbf{x})=\frac{s \sin \left(\left\{\left|\Phi^{(0)}\right|\right\} \pi\right)}{\pi^{3} r^{2}} \int_{0}^{\infty} d w \frac{K_{\left.\left\{\mid \Phi^{(0)}\right\}\right\}}(w) K_{1-\left\{\Phi^{(0)}\right\}}(w)}{\cosh \left[\left(2\left\{\left|\Phi^{(0)}\right|\right\}-1\right) \ln \left(\frac{w}{\mu r}\right)+\ln \bar{A}\right]} .
$$

Consequently, we get the following expression for vacuum condensate (10):

$$
\mathcal{P}(\mathbf{x})=-\frac{s \sin \left(\left\{\left|\Phi^{(0)}\right|\right\} \pi\right)}{\pi^{3} r^{2}} \int_{0}^{\infty} d w w \frac{K_{\left\{\Phi^{(0)} \mid\right\}}^{2}(w)+K_{1-\left\{\Phi^{(0)}\right\}}^{2}(w)}{\cosh \left[\left(2\left\{\left|\Phi^{(0)}\right|\right\}-1\right) \ln \left(\frac{w}{\mu r}\right)+\ln \bar{A}\right]} .
$$


As well as in the case considered in the previous section, energy density (70) and current (71) are also induced in the vacuum. However, there are additional vacuum polarization effects in the case of the chiral invariant condition (38).

Let us define vacuum fermion number density

$$
\mathcal{N}(\mathbf{x})=-\frac{1}{2} \operatorname{tr}\langle\mathbf{x}|\operatorname{sgn}(H)| \mathbf{x}\rangle,
$$

vacuum spin density

$$
\mathcal{S}(\mathbf{x})=\frac{1}{8 i} \operatorname{tr}\left\langle\mathbf{x}\left|\left[\gamma^{1}, \gamma^{2}\right]_{-} \operatorname{sgn}(H)\right| \mathbf{x}\right\rangle
$$

and vacuum angular momentum density

$$
\mathcal{M}(\mathbf{x})=\frac{i}{2} \operatorname{tr}\left\langle\mathbf{x}\left|\left(\mathbf{x} \times \boldsymbol{\partial}-\frac{1}{4}\left[\gamma^{1}, \gamma^{2}\right]_{-}\right) \operatorname{sgn}(H)\right| \mathbf{x}\right\rangle .
$$

As it has been already noted in Introduction, the vacuum spin density is directly related to the parity breaking vacuum condensate:

$$
\mathcal{S}(\mathbf{x})=\frac{1}{2} \mathcal{P}(\mathbf{x})
$$

As to remaining vacuum densities (82) and (84), in background (14)-(15) under condition (38), they take form

$$
\mathcal{N}(\mathbf{x})=-\frac{s \sin \left(\left\{\left|\Phi^{(0)}\right|\right\} \pi\right)}{\pi^{3} r^{2}} \int_{0}^{\infty} d w w \frac{K_{\left\{\left|\Phi^{(0)}\right|\right\}}^{2}(w)-K_{1-\left\{\mid \Phi^{(0)}\right\}}^{2}(w)}{\cosh \left[\left(2\left\{\left|\Phi^{(0)}\right|\right\}-1\right) \ln \left(\frac{w}{\mu r}\right)+\ln \bar{A}\right]}
$$

and

$$
\mathcal{M}(\mathbf{x})=\left(\left[\left[\Phi^{(0)}\right]\right]+\frac{1}{2}\right) \mathcal{N}(\mathbf{x}) .
$$

Thus, contrary to the parity breaking condensate ,

$$
\left.\mathcal{P}(\mathbf{x})\right|_{\left\{\Phi^{(0)}\right\}=\frac{1}{2}}=-\frac{s \cos \Theta}{2 \pi^{2} r^{2}},
$$

the vacuum fermion number and angular momentum vanish at half-integer values of the vortex flux. At large distances from the vortex, we get

$$
\mathcal{N}(\mathbf{x}) \underset{r \rightarrow \infty}{=}-\left(\left\{\left|\Phi^{(0)}\right|\right\}-\frac{1}{2}\right) \frac{\sin \left(\left\{\left|\Phi^{(0)}\right|\right\} \pi\right)}{\pi^{2} r^{2}} \times
$$




$$
\times\left\{\begin{array}{cl}
(\mu r)^{2\left\{\mid \Phi^{(0)}\right\}-1} \bar{A}^{-1} \frac{\Gamma\left(\frac{3}{2}-\left\{\mid \Phi^{(0)}\right\}\right) \Gamma\left(\frac{3}{2}-2\left\{\mid \Phi^{(0)}\right\}\right)}{\left.\Gamma\left(2-\left\{\Phi^{(0)}\right\}\right\}\right)}, & 0<\left\{\left|\Phi^{(0)}\right|\right\}<\frac{1}{2} \\
(\mu r)^{1-2\left\{\mid \Phi^{(0)}\right\}} \bar{A} \frac{\left.\Gamma\left(\left\{\Phi^{(0)}\right\}+\frac{1}{2}\right) \Gamma\left(2\left\{\Phi^{(0)}\right\}\right\}-\frac{1}{2}\right)}{\Gamma\left(1+\left\{\Phi^{(0)}\right\}\right)}, & \frac{1}{2}<\left\{\left|\Phi^{(0)}\right|\right\}<1
\end{array} .\right.
$$

Finally, defining the global vacuum characteristics,

$$
\mathcal{P}=\int d^{2} x \mathcal{P}(\mathbf{x}), \mathcal{N}=\int d^{2} x \mathcal{N}(\mathbf{x}), \mathcal{M}=\int d^{2} x \mathcal{M}(\mathbf{x})
$$

we get

$$
\begin{gathered}
\mathcal{P}=-\frac{s \operatorname{sgn}(\cos \Theta)}{\left|2\left\{\left|\Phi^{(0)}\right|\right\}-1\right|} \\
\mathcal{N}=-\frac{1}{2} s \operatorname{sgn}\left[\left(\left\{\left|\Phi^{(0)}\right|\right\}-\frac{1}{2}\right) \cos \Theta\right] \\
\mathcal{M}=-\frac{1}{2} s\left(\left[\left[\Phi^{(0)}\right]\right]+\frac{1}{2}\right) \operatorname{sgn}\left[\left(\left\{\left|\Phi^{(0)}\right|\right\}-\frac{1}{2}\right) \cos \Theta\right] .
\end{gathered}
$$

\section{Absence of twodimensional anomaly}

Omitting the time dimension (i.e. putting $x^{0}=$ const), let us define twodimensional Euclidean effective action functional

$$
S^{\mathrm{eff}}[\mathbf{V}(\mathbf{x})]=-\ln \int d \Psi d \Psi^{\dagger} \exp \left(-\int d^{2} x \Psi^{\dagger} H \Psi\right)=-\ln \operatorname{det} H
$$

The last equation is formally invariant under transformation

$$
\Psi \rightarrow e^{i \omega \Gamma} \Psi, \quad \Psi^{\dagger} \rightarrow \Psi^{\dagger} e^{i \omega \Gamma}
$$

where $\Gamma$ is a matrix which anticommutes with the Hamiltonian,

$$
[H, \Gamma]_{+}=0, \quad \operatorname{tr} \Gamma=0, \quad \Gamma^{2}=I .
$$

The invariance under the localized (coordinate dependent) generalization of transformation (95) corresponds to the conservation law:

$$
\boldsymbol{\nabla} \cdot \mathbf{J}^{3}(\mathbf{x})=0
$$

where

$$
\mathbf{J}^{3}(\mathbf{x})=i \operatorname{tr}\left\langle\mathbf{x}\left|\gamma^{0} \gamma \Gamma H^{-1}\right| \mathbf{x}\right\rangle
$$


However, both functional (94) and current (98) are ill-defined, suffering from ultraviolet as well as infrared divergences. Performing the regularization of divergences with the use of the zeta function method [21, 22, 23], one arrives at, instead of Eq.(97), the following relation:

$$
\boldsymbol{\nabla} \cdot \mathbf{J}^{3}(\mathbf{x})=2 \lim _{z \rightarrow 0} \lim _{M \rightarrow 0} \tilde{\zeta}_{\mathbf{x}}(z \mid M)
$$

where

$$
\tilde{\zeta}_{\mathbf{x}}(z \mid M)=\operatorname{tr}\left\langle\mathbf{x}\left|\Gamma\left(H^{2}+M^{2}\right)^{-z}\right| \mathbf{x}\right\rangle
$$

is the modified zeta function density (compare with Eq.(65)).

In the reducible $4 \times 4$ representation of the Clifford algebra (see Eq.(21)), the role of $\Gamma$ can be played by each of the following four matrices:

$$
\gamma^{0}, \quad \gamma^{0} \gamma^{3}, \quad \gamma^{0} \gamma^{5}, \quad \frac{1}{2} \gamma^{0}\left[\gamma^{3}, \gamma^{5}\right]_{-}
$$

However, only one choice,

$$
\Gamma=\frac{1}{2} \gamma^{0}\left[\gamma^{3}, \gamma^{5}\right]_{-}
$$

can lead to the nonvanishing modified zeta function density and, thus, to the anomaly in the conservation of the appropriate current,

$$
\mathbf{J}^{3}(\mathbf{x})=\frac{1}{2 i} \operatorname{tr}\left\langle\mathbf{x}\left|\gamma\left[\gamma^{3}, \gamma^{5}\right]_{-} H^{-1}\right| \mathbf{x}\right\rangle
$$

Reflecting a generic relationship between Eq.(102) and current $\mathcal{I}(\mathbf{x})$ (11), this anomaly is usually called as the parity anomaly.

The question that we would like to address in the present section is: whether singular magnetic vortex (14)-(15) induces the parity anomaly or not? It is clear that the parity anomaly is absent under the parity invariant condition (40). So there remains to check the absence of the parity anomaly under the chiral invariant condition (38). Similarly to that in the above sections, we find that

$$
\tilde{\zeta}_{\mathbf{x}}(z \mid M)=\frac{1}{2} \operatorname{tr}\left\langle\mathbf{x}\left|\gamma^{0}\left[\gamma^{3}, \gamma^{5}\right]_{-}\left(H^{2}+M^{2}\right)^{-z}\right| \mathbf{x}\right\rangle
$$

under condition (38) takes form

$$
\tilde{\zeta}_{\mathbf{x}}(z \mid M)=\frac{2 s \sin \left(\left\{\left|\Phi^{(0)}\right|\right\} \pi\right)}{\pi^{3}} \sin (z \pi) r^{2(z-1)} \times
$$




$$
\begin{gathered}
\times \int_{|M| r}^{\infty} d w w\left(w^{2}-M^{2} r^{2}\right)^{-z}\left\{K_{\left.\left\{\Phi^{(0)}\right\}\right\}}^{2}(w)-K_{1-\left\{\Phi^{(0)}\right\}}^{2}(w)+\right. \\
\left.+\left[K_{\left\{\Phi^{(0)}\right\}}^{2}(w)+K_{\left.1-\left\{\Phi^{(0)}\right\}\right\}}^{2}(w)\right] \tanh \left[\left(2\left\{\left|\Phi^{(0)}\right|\right\}-1\right) \ln \left(\frac{w}{\mu r}\right)+\ln \bar{A}\right]\right\} .
\end{gathered}
$$

Hence, one obtains immediately

$$
\lim _{z \rightarrow 0} \tilde{\zeta}_{\mathbf{x}}(z \mid M)=0 \quad(M \neq 0)
$$

It is more instructive to take limit $M \rightarrow 0$ first and then to consider limit $z \rightarrow 0$. Thus, we get

$$
\begin{aligned}
& \tilde{\zeta}_{\mathbf{x}}(z \mid 0)=\frac{s \sin \left(\left\{\left|\Phi^{(0)}\right|\right\} \pi\right)}{\pi^{2}} r^{2(z-1)}\left\{\frac{\sqrt{\pi}\left(\left\{\left|\Phi^{(0)}\right|\right\}-\frac{1}{2}\right)}{\Gamma(z) \Gamma\left(\frac{3}{2}-z\right)} \Gamma\left(\left\{\left|\Phi^{(0)}\right|\right\}-z\right) \Gamma\left(1-\left\{\left|\Phi^{(0)}\right|\right\}-z\right)+\right. \\
& \left.+\frac{2 \sin (z \pi)}{\pi} \int_{0}^{\infty} d w w^{1-2 z}\left[K_{\left\{\mid \Phi^{(0)}\right\}}^{2}(w)+K_{1-\left\{\Phi^{(0)}\right\}}^{2}(w)\right] \tanh \left[\left(2\left\{\left|\Phi^{(0)}\right|\right\}-1\right) \ln \left(\frac{w}{\mu r}\right)+\ln \bar{A}\right]\right\} ;
\end{aligned}
$$

in particular, at half-integer values of the vortex flux:

$$
\left.\tilde{\zeta}_{\mathbf{x}}(z \mid 0)\right|_{\left.\left\{\Phi^{(0)}\right\}\right\}=\frac{1}{2}}=\frac{s \sin \Theta}{\pi^{\frac{3}{2}}} \frac{\Gamma\left(\frac{1}{2}-z\right)}{\Gamma(z)} r^{2(z-1)} ;
$$

and in the case when Eq.(43) holds:

$$
\begin{gathered}
\tilde{\zeta}_{\mathbf{x}}(z \mid 0)= \pm \frac{s \sin \left(\left\{\left|\Phi^{(0)}\right|\right\} \pi\right)}{\pi^{\frac{3}{2}}} \times \\
\times \frac{\Gamma\left(\frac{3}{2}-z \pm\left\{\left|\Phi^{(0)}\right|\right\} \mp \frac{1}{2}\right) \Gamma\left(\frac{1}{2}-z \mp\left\{\left|\Phi^{(0)}\right|\right\} \pm \frac{1}{2}\right)}{\Gamma(z) \Gamma\left(\frac{3}{2}-z\right)} r^{2(z-1)}, \quad \Theta= \pm s \frac{\pi}{2}
\end{gathered}
$$

Consequently, we obtain

$$
\tilde{\zeta}_{\mathbf{x}}(0 \mid 0)=0, \quad \mathbf{x} \neq 0
$$

which ensures the validity of Eq.(97) everywhere on the plane with the puncture at $\mathbf{x}=0$.

\section{Conclusion}

Before discussing our results, let us recall the well-known fact: a regular configuration of external magnetic field strength $\boldsymbol{\partial} \times \mathbf{V}(\mathbf{x})$ in 2+1-dimensional space-time 
induces the parity anomaly [24, 25, 26],

$$
\boldsymbol{\nabla} \cdot \mathbf{J}^{3}(\mathbf{x})=\frac{2 s}{\pi} \boldsymbol{\partial} \times \mathbf{V}(\mathbf{x})
$$

as well as the chiral symmetry breaking vacuum condensate [5],

$$
\mathcal{C}(\mathbf{x})=-\frac{1}{2 \pi}|\partial \times \mathbf{V}(\mathbf{x})|
$$

These relations exhibit a direct (or local) impact of an external field strength on a quantized fermion field. If one excludes the spatial region of nonvanishing field strength and imposes a physically sensible condition at the boundary of the excluded region, what happens then with a quantized fermion field in the remaining part of space? Basing merely on Eqs.(110) and (111), one could expect that both the anomaly and the condensate vanish in the region of vanishing field strength. However, as it is shown in the present paper, these naive expectations are justified for the anomaly only (see Section 5), whereas the condensate and other vacuum quantum numbers appear to be nonvanishing, thus exhibiting an indirect (or nonlocal) impact of an external field strength, which may be regarded (see, e.g., Ref. 14]) as a leak through the boundary of the excluded region.

To be more precise, we consider the situation when the volume of the excluded region is shrunk to zero, while the global characteristics of an external field strength (flux) is retained nonvanishing. Therefore, singular magnetic vortex (14)-(15) is taken as an external field configuration. The boundary condition at the location of the vortex has to ensure self-adjointness of the Hamiltonian. We find two sets of boundary conditions - one is chiral invariant (38) and another is parity invariant (40); each set is labelled by the self-adjoint extension parameter. Thus, vacuum polarization effects in the background of a singular magnetic vortex are depending both on the vortex flux and the self-adjoint extension parameter.

As it should be expected, the vacuum polarization effects remain invariant under the transition to the equivalent representation of the Clifford algebra (i.e. do not depend on $\chi_{ \pm}$). If the parity invariant condition (40) is imposed, then the vacuum polarization effects are invariant under the transition to the inequivalent representation of the Clifford algebra (i.e. do not depend on $s$ ). They comprise chiral 
symmetry breaking vacuum condensate (56), vacuum energy density (70) and vacuum current (71). If the chiral invariant condition (38) is imposed, then the vacuum polarization effects are either invariant or changing sign under $s \rightarrow-s$. The invariant effects comprise vacuum energy density (70) and vacuum current (71), while the changing sign effects comprise parity breaking vacuum condensate (81), vacuum fermion number density (86) and vacuum angular momentum density (87). All vacuum polarization effects are decreasing as inverse powers (with integer exponents in the cases of vacuum energy density and current, and with fractional exponents otherwise) at large distances from the vortex, see Eqs.(74)-(76) and (89). Total vacuum condensates are finite at non-half-integer values of the vortex flux, see Eqs.(63) and (91). Total vacuum fermion number and angular momentum are finite, vanishing at half-integer values of the vortex flux, see Eqs.(92) and (93).

It should be noted that both conditions (38) and (40) become scale invariant at half-integer values of the vortex flux. Thus, Eq.(58) exhibits a scale invariant pattern of chiral symmetry breaking. Other scale invariant effects are given by vacuum energy density (72), vacuum current (73) and parity breaking vacuum condensate (88). The infinite value of total vacuum condensates $(63)$ and $(91)$ at $\left\{\left|\Phi^{(0)}\right|\right\}=\frac{1}{2}$ is a consequence of scale invariance.

Finally, let us discuss the case of Eq.(43) when conditions (38) and (40) coincide. A distinctive feature in this case is that two of the four components of wave function (31) become regular for all $n$ : if $\Theta=s \frac{\pi}{2}$, then the $g_{n}^{ \pm}$components are regular, and, if $\Theta=-s \frac{\pi}{2}$, then the $f_{n}^{ \pm}$components are regular. Parity and chiral symmetry, as well as scale symmetry, are conserved, and only energy density and current are induced in the vacuum:

$$
\begin{gathered}
\varepsilon^{\mathrm{ren}}(\mathbf{x})=\frac{\tan (\{|\Phi|\} \pi)}{2 \pi r^{3}}\left(\{|\Phi|\}-\frac{1}{2}\right)\left[\frac{1}{3}\{|\Phi|\}(1-\{|\Phi|\})-\frac{1}{4} \mp \frac{1}{2}\left(\{|\Phi|\}-\frac{1}{2}\right)\right], \\
\Theta= \pm \frac{\pi}{2}, \\
j_{\varphi}(\mathbf{x})=\frac{\tan (\{|\Phi|\} \pi)}{2 \pi r^{2}}\left(\{|\Phi|\}-\frac{1}{2}\right)\left(\{|\Phi|\}-\frac{1}{2} \pm 1\right), \quad \Theta= \pm \frac{\pi}{2} .
\end{gathered}
$$




\section{Acknowledgements}

I am grateful to H. Leutwyler, V.A. Miransky and W. Thirring for stimulating discussions and interesting remarks. The research was supported by the State Foundation for Fundamental Research of Ukraine (project 2.4/320).

\section{Appendix A}

The partial Hamiltonian corresponding to $n=n_{0}$ takes form

$$
h=\left(\begin{array}{cc}
h_{+} & 0 \\
0 & h_{-}
\end{array}\right)
$$

where (see Eq.(32))

$$
h_{ \pm}=\left(\begin{array}{cc}
0 & e^{i \chi_{ \pm}\left[\partial_{r}+(1-F) r^{-1}\right]} \\
e^{-i \chi_{ \pm}}\left(-\partial_{r}-F r^{-1}\right) & 0
\end{array}\right) .
$$

Let $h$ be the operator in the form of Eqs.(A.1)-(A.2), which acts on the domain of functions $\xi^{(0)}(r)$ that are regular at $r=0$. Then its adjoint $h^{\dagger}$ which is defined by relation

$$
\int_{0}^{\infty} d r r\left[h^{\dagger} \xi(r)\right]^{\dagger} \xi^{(0)}(r)=\int_{0}^{\infty} d r r[\xi(r)]^{\dagger}\left[h \xi^{(0)}(r)\right]
$$

acts on the domain of functions $\xi(r)$ that are not necessarily regular at $r=0$. So the question is, whether the domain of definition of $h$ can be extended, resulting in both the operator and its adjoint being defined on the same domain of functions? To answer this, one has to construct the eigenspaces of $h^{\dagger}$ with complex eigenvalues. They are spanned by the linearly independent square-integrable solutions correspoding to the pair of purely imaginary eigenvalues,

$$
h^{\dagger} \xi^{(1)}(r)=i \mu \xi^{(1)}(r), \quad h^{\dagger} \xi^{(2)}(r)=-i \mu \xi^{(2)}(r),
$$

where $\mu>0$ is inserted merely for the dimension reasons. It can be shown that, in the case of Eqs.(A.1)-(A.2), only one pair of such solutions exists:

$$
\xi^{(1)}(r)=\left(\begin{array}{c}
\xi_{+}^{(1)}(r) \\
\xi_{-}^{(1)}(r)
\end{array}\right), \quad \xi^{(2)}(r)=\left(\begin{array}{c}
\xi_{+}^{(2)}(r) \\
\xi_{-}^{(2)}(r)
\end{array}\right)
$$


where

$$
\xi_{ \pm}^{(1)}(r)=\frac{1}{N}\left(\begin{array}{c}
e^{i \chi_{ \pm}} e^{i \frac{\pi}{4}} K_{F}(\mu r) \\
e^{-i \frac{\pi}{4}} K_{1-F}(\mu r)
\end{array}\right), \quad \xi_{ \pm}^{(2)}(r)=\frac{1}{N}\left(\begin{array}{c}
e^{i \chi_{ \pm}} e^{-i \frac{\pi}{4}} K_{F}(\mu r) \\
e^{i \frac{\pi}{4}} K_{1-F}(\mu r)
\end{array}\right)
$$

$N$ is a certain normalization factor. Thus the deficiency index of each of operators $h_{+}$and $h_{-}$is equal to $(1,1)$. Then, according to the Weyl - von Neumann theory of self-adjoint operators (see Refs. [10, 11]), the self-adjoint extension of operator $h_{ \pm}$is defined on the domain of functions of the following form

$$
\left(\begin{array}{c}
f_{n_{0}}^{ \pm} \\
g_{n_{0}}^{ \pm}
\end{array}\right)=\xi_{ \pm}^{(0)}+c\left(\xi_{ \pm}^{(1)}+e^{i \theta_{ \pm}(s)} \xi_{ \pm}^{(2)}\right)
$$

where $c$ is a complex constant and $\theta_{ \pm}(s)$ is a real continuous parameter which depends on $s$. Using the asymptotics of the Macdonald function at small values of the variable, we find that operator (A.1)-(A.2) is self-adjoint when defined on the domain of functions with the following asymptotic behaviour:

$$
\left(\begin{array}{c}
f_{n_{0}}^{+} \\
g_{n_{0}}^{+} \\
f_{n_{0}}^{-} \\
g_{n_{0}}^{-}
\end{array}\right) \underset{r \rightarrow 0}{\sim}\left(\begin{array}{c}
e^{i \chi_{+}} \sin \left[\frac{1}{2} \theta_{+}(s)+\frac{\pi}{4}\right] 2^{F} \Gamma(F)(\mu r)^{-F} \\
\cos \left[\frac{1}{2} \theta_{+}(s)+\frac{\pi}{4}\right] 2^{1-F} \Gamma(1-F)(\mu r)^{-1+F} \\
e^{i \chi_{-}} \sin \left[\frac{1}{2} \theta_{-}(s)+\frac{\pi}{4}\right] 2^{F} \Gamma(F)(\mu r)^{-F} \\
\cos \left[\frac{1}{2} \theta_{-}(s)+\frac{\pi}{4}\right] 2^{1-F} \Gamma(1-F)(\mu r)^{-1+F}
\end{array}\right) .
$$

If one chooses

$$
\theta_{+}(s)=\theta_{-}(s)=s \theta
$$

then asymptotics (A.8) is invariant under the chiral symmetry transformation (25) and the following relation holds

$$
\cos \left(s \frac{\theta}{2}+\frac{\pi}{4}\right) 2^{1-F} \Gamma(1-F) \lim _{r \rightarrow 0}(\mu r)^{F} f_{n_{0}}^{ \pm}=e^{i \chi_{ \pm}} \sin \left(s \frac{\theta}{2}+\frac{\pi}{4}\right) 2^{F} \Gamma(F) \lim _{r \rightarrow 0}(\mu r)^{1-F} g_{n_{0}}^{ \pm} .
$$

If one chooses

$$
\theta_{+}(s)=s \theta, \quad \theta_{-}(s)=-s \theta+\pi,
$$

then asymptotics (A.8) is invariant under the parity transformation (24) and the following relation holds

$$
\cos \left(s \frac{\theta}{2}+\frac{\pi}{4}\right) 2^{1-F} \Gamma(1-F) \lim _{r \rightarrow 0}(\mu r)^{F} f_{n_{0}}^{ \pm}= \pm e^{i \chi_{ \pm}} \sin \left(s \frac{\theta}{2}+\frac{\pi}{4}\right) 2^{F} \Gamma(F) \lim _{r \rightarrow 0}(\mu r)^{1-F} g_{n_{0}}^{ \pm} .
$$


Defining new parameter $\Theta$ by means of relation

$$
\tan \left(s \frac{\Theta}{2}+\frac{\pi}{4}\right)=-2^{2 F-1} \frac{\Gamma(F)}{\Gamma(1-F)} \tan \left(s \frac{\theta}{2}+\frac{\pi}{4}\right)
$$

we rewrite Eqs.(A.10) and (A.12) in the form of Eqs.(38) and (40) respectively.

\section{Appendix B}

Inserting regulator mass $M$ into Eq.(2), we get

$$
\langle\operatorname{vac}|T \Psi(x) \bar{\Psi}(y)| \operatorname{vac}\rangle=-i\left\langle x\left|\left(-i \gamma^{\mu} \nabla_{\mu}+M\right)^{-1}\right| y\right\rangle
$$

Then the regularized condensate takes form

$$
\begin{aligned}
& \mathcal{C}(\mathbf{x} \mid M)=-\frac{1}{2} \operatorname{tr}\left\langle\mathbf{x}\left|\gamma^{0} \operatorname{sgn}\left(H+\gamma^{0} M\right)\right| \mathbf{x}\right\rangle= \\
& =\frac{i}{4} \nabla \cdot \operatorname{tr}\left\langle\mathbf{x}\left|\gamma\left(H^{2}+M^{2}\right)^{-\frac{1}{2}}\right| \mathbf{x}\right\rangle-\frac{1}{2} M \zeta_{\mathbf{x}}\left(\frac{1}{2} \mid M\right),
\end{aligned}
$$

where zeta function density $\zeta_{\mathbf{x}}(z \mid M)$ is defined by Eq.(65). Defining regularized current

$$
\mathcal{J}(\mathbf{x} \mid M)=\frac{i}{4} \operatorname{tr}\left\langle\mathbf{x}\left|\boldsymbol{\gamma}\left(H^{2}+M^{2}\right)^{-\frac{1}{2}}\right| \mathbf{x}\right\rangle
$$

we get

$$
\begin{aligned}
\mathcal{J}_{r}(\mathbf{x} \mid M) & =\frac{\sin \left(\left\{\left|\Phi^{(0)}\right|\right\} \pi\right)}{\pi^{3} r^{2}} \int_{|M| r}^{\infty} d w \frac{w}{\sqrt{w^{2}-M^{2} r^{2}}} \times \\
& \times \frac{K_{\left\{\Phi^{(0)}\right\}}(w) K_{1-\left\{\Phi^{(0)}\right\}}(w)}{\cosh \left[\left(2\left\{\Phi^{(0)} \mid\right\}-1\right) \ln \left(\frac{w}{\mu r}\right)+\ln \bar{A}\right]},
\end{aligned}
$$

and

$$
\mathcal{J}_{\varphi}(\mathbf{x} \mid M)=0
$$

consequently,

$$
\boldsymbol{\nabla} \cdot \mathcal{J}(\mathbf{x} \mid M)=-\frac{\sin \left(\left\{\left|\Phi^{(0)}\right|\right\} \pi\right)}{\pi^{3} r^{2}} \int_{|M| r}^{\infty} d w \frac{w^{2}}{\sqrt{w^{2}-M^{2} r^{2}}} \times
$$




$$
\times \frac{K_{\left\{\Phi^{(0)}\right\}}^{2}(w)+K_{1-\left\{\Phi^{(0)}\right\}}^{2}(w)}{\cosh \left[\left(2\left\{\left\{\Phi^{(0)} \mid\right\}-1\right) \ln \left(\frac{w}{\mu r}\right)+\ln \bar{A}\right]\right.} .
$$

In particular, at half-integer values of the vortex flux (at $\left\{\Phi^{(0)} \mid\right\}=\frac{1}{2}$ ) we get

$$
\left.\mathcal{J}_{r}(\mathbf{x} \mid M)\right|_{\left\{\Phi{ }^{(0)}\right\}=\frac{1}{2}}=\frac{\cos \Theta}{2 \pi^{2} r} K_{0}(2|M| r)
$$

and

$$
\left.\boldsymbol{\nabla} \cdot \mathcal{J}(\mathbf{x} \mid M)\right|_{\left\{\Phi^{(0)}\right\}=\frac{1}{2}}=-\frac{\cos \Theta}{\pi^{2} r}|M| K_{1}(2|M| r) ;
$$

consequently,

$$
\left.\mathcal{J}_{r}(\mathbf{x} \mid M \rightarrow 0)\right|_{\left\{\Phi^{(0)}\right\}=\frac{1}{2}}=-\frac{\cos \Theta}{2 \pi^{2} r} \ln (2|M| r)
$$

and

$$
\left.\boldsymbol{\nabla} \cdot \mathcal{J}(\mathbf{x} \mid M \rightarrow 0)\right|_{\left\{\Phi^{(0)}\right\}=\frac{1}{2}}=-\frac{\cos \Theta}{2 \pi^{2} r^{2}} .
$$

Using Eq.(69), we get relation

$$
\begin{aligned}
& \zeta_{\mathbf{x}}\left(\frac{1}{2} \mid M\right)=-\frac{2}{\pi}|M|-\frac{8 \sin \left(\left\{\Phi^{(0)}\right\} \pi\right)}{\pi^{3} r} \int_{|M| r}^{\infty} d w \sqrt{w^{2}-M^{2} r^{2}} K_{\left\{\Phi^{(0)}\right\}}(w) K_{1-\left\{\Phi^{(0)}\right\}}(w)+ \\
& +\frac{\left.2 \sin \left(\left\{\mid \Phi^{(0)}\right\}\right\} \pi\right)}{\pi^{3} r} \int_{|M| r}^{\infty} d w \frac{w}{\sqrt{w^{2}-M^{2} r^{2}}}\left\{K_{\left\{\Phi^{(0)}\right\}}^{2}(w)+K_{1-\left\{\mid \Phi^{(0)}\right\}}^{2}(w)+\right. \\
& \left.\left.+\left[K_{\left\{\Phi^{(0)}\{\}\right.}^{2}(w)-K_{1-\left\{\Phi^{(0)}\right\}}^{2}(w)\right] \tanh \left[\left(2\left\{\mid \Phi^{(0)}\right\}\right\}-1\right) \ln \left(\frac{w}{\mu r}\right)+\ln \bar{A}\right]\right\},
\end{aligned}
$$

and, consequently,

$$
\lim _{M \rightarrow 0} M \zeta_{\mathbf{x}}\left(\frac{1}{2} \mid M\right)=0 .
$$

Thus, Eqs.(B.4) and (B.6) in the limit of $M \rightarrow 0$ yield Eqs.(53) and (56), respectively, and Eq.(B.10) coincides with Eq.(58). Note also relations

$$
\left.\lim _{r \rightarrow \infty} r \mathcal{J}_{r}(\mathbf{x} \mid M)\right|_{\left\{\Phi^{(0)}\right\}=\frac{1}{2}}=0 \quad(M \neq 0)
$$

and

$$
\left.\operatorname{sgn}(\cos \Theta) \lim _{r \rightarrow 0} r \mathcal{J}_{r}(\mathbf{x} \mid M)\right|_{\left\{\Phi^{(0)}\right\}=\frac{1}{2}}=\infty \quad\left(\Theta \neq \pm \frac{\pi}{2}\right) ;
$$

thus, the total condensate at half-integer values of the vortex flux is infinite even at finite values of $M$. 


\section{References}

[1] G.W. Semenoff and L.C.R. Wijewardhana, Phys. Rev. D45, 1342 (1992).

[2] N. Dorey and N.E. Mavromatos, Nucl.Phys. B386, 614 (1992); N.E. Mavromatos, Nucl.Phys. (Proc. Suppl.) C33, 145 (1993).

[3] R. Mackenzie, P.K. Panigrakhi and S. Sakhi, Phys.Rev. B48, 3892 (1993).

[4] K. Farakos, G. Koutsoumbas, N.E. Mavromatos and A. Momen, "On magnetic catalysis in even-flavor $Q E D_{3}$ ", preprint CERN-TH-99-123 (May 1999); hep$\mathrm{ph} / 9905272$.

[5] V.P. Gusynin, V.A. Miransky and I.A. Shovkovy, Phys. Rev. Lett. 73, 3499 (1994); Phys. Rev. D52, 4718 (1995).

[6] G. Dunne and T. Hall, Phys.Rev. D53, 2220 (1996).

[7] Yu.A. Sitenko, Mod. Phys. Lett. A14, 701 (1999).

[8] Y. Aharonov and D. Bohm, Phys. Rev. 115, 485 (1959).

[9] T.W. Appelquist, M. Bowick, D. Karabali and L.C.R. Wijewardhana, Phys. Rev. D33, 3704 (1986).

[10] N.I. Akhiezer and I.M. Glazman, Theory of Linear Operators in Hilbert Space (Pitman, Boston, 1981) V.2.

[11] S. Albeverio, F. Gesztesy, R. Hoegh-Krohn and H. Holden, Solvable Models in Quantum Mechanics (Springer-Verlag, Berlin, 1988).

[12] A.S. Goldhaber, Phys.Rev. D16, 1815 (1977).

[13] C.J. Callias, Phys.Rev. D16, 3068 (1977).

[14] B. Grossman, Phys.Rev.Lett. 50, 464 (1983).

[15] H. Yamagishi, Phys.Rev. D27, 2383; D28, 977 (1983). 
[16] Ph. de S. Gerbert, Phys. Rev. D40, 1346 (1989).

[17] Yu.A. Sitenko, Phys. Lett. B387, 334 (1996).

[18] Yu.A. Sitenko, Phys. Atom. Nucl. 60, 2102 (1997).

[19] Yu.A. Sitenko, Phys. Atom. Nucl. 62, 1056 (1999).

[20] P.A.M. Dirac, Proc. Roy. Soc. (London) A133, 60 (1931).

[21] A. Salam and J. Strathdee, Nucl. Phys. B90, 203 (1975).

[22] J.S. Dowker and R. Critchley, Phys. Rev. D13, 3224 (1976).

[23] S. Hawking, Commun. Math. Phys. 55, 133 (1977).

[24] J. Schwinger, Phys.Rev. 128, 2425 (1962).

[25] S.-S. Shei, Phys.Rev. D6, 3469 (1972).

[26] R. Jackiw, Dynamical symmetry breaking, in Laws of Hadronic Matter, edited by A.Zichichi (Academic Press, New York, 1975). 